smaller or finer particles. Moist dust was also found to be more difficult to sterilize than when in the dry state, all of which tends to illustrate, to a certain extent, the penetrative power of formaldehyde.

The foregoing personal experiments seem to confine success in sterilization to superficial objects. Wher. ever an attempt was made at concealment of any degree, the result was in all instances negative. This agrees with the experience of Trillat and G. Roux, Bosc, ${ }^{5}$ Vaillard and Lemoine, ${ }^{6}$ Pfuhl $^{7}$ and Ascoli. The results obtained are commensurate with the size of lamp or apparatus, that is, the amount of aldehyde gas generated and the size of the room. As to the amount of gas necessary for thorough sterilization this seems rather difficult to determine with any degree of exactness.

In generating the gas from methyl alcohol. Pfuhl has estimated the amount of wood alcohol to be used for every cubic meter as follows: bacillus of typhoid, sterilized by 47.1 grams; bacillus of diphtheria, sterilized by 21.2 grams; staphylococcus pyognes aureus, sterilized by 62.8 grams; bacillus of anthrax spores, not by 94.1 grams; bacillus of tetanus spores, not by 94.1 grams. Taking these figures as a guide it would require not less than 2 liters of wood alcohol for 1000 cubic feet of space.

In the autoclave method where the 40 per cont. formaldehyde solution is used, Paul Struver ${ }^{8}$ places the required amount at 8.2 centimeters of formaldehyde ( 40 per cent.) for each cubic meter (35.28 cubic feet), while the Marine-Hospital Service Circular No. 121 fixes the same at 14 . c.c. per cubic meter. With these standards accepted the negative results obtained in the above named tests would be explained.

The conclusions naturally drawn from the results must be that small generators or lamps, such as the No. 1 Hollister and others of similar type, are not adapted for room sterilization, and should be confined to use in very small areas of space. The No. 2 generator, having a capacity of 4 quarts of methyl alco. hol, should only be used for room sterilization, and then I would place the limit at 2000 to 2500 cubic feet; if larger space is to be disinfected, more lamps should be added in proportion.

Articles in the room should be exposed freely, clothing spread about, pockets turned inside out, and all concealment avoided as much as possible.

The cheapness and the little attention required in producing formaldehyde gas from wood alcohol, renders this method very desirable, but the use of the several forms of apparatus so far proposed is attended by quite a number of defects, which are mainly due to the fact that the slow conversion of the alcohol allows the gas to escape through the many crevices and openings necessarily found in every room, before it has had an opportunity to penetrate into the depths of substances contained in the apartment. The latter difficulty is overcome to a certain extent by the auto. clave method of generating the gas; the reports of its use are certainly more promising. The rather high cost of the apparatus will interfere somewhat with its general introduction, but by this method the gas escapes under a high pressure and in corresponding larger amount, the diffusion is more rapid and sterilization, in consequence, more complete.

In the recent able article of Doty, on "Disinfection by Steam," the virtues of this agent are brought forward most forcibly, corroborating what has been known heretofore of its effective power when used on a smaller scale; and although here, also, expense enters as an important item, there can be no question that in penetrative power, rapidity of action and especially in the sterilization of lineu, blankets, bedding, etc, steam can not be displaced by formaldehyde. All observers concede the superiority of formaldehyde gas over sulphurous oxid for room disinfection.

As a sterilizing agent for surgical purposes, an alcohol gas generator may be used, when confined to space not exceeding two and one-half cubic feet, and used for instruments only, with an exposure of at least thirty minutes, for I question the practicability of its use for sterilizing sheets, towels, gauzes or dressings of any kind. Used in solutions of 1 to 200 , to 1 to 500 it forms a most efficient agent for the disinfection of instruments, and is superior to carbolic acid or corrosive sublimate, having no injurious effects, nor is it disagreeable to the hands of the operator. As a deodorant and disinfectant for general purposes, when used in solution, it has no equal. REFERENCES.

Zeitschrift f. Hygiene u. Iniections Kr., Vol. XXI, p. 421

Public Health Reports, Vol. XII, No. 5 .

$, 4,5$ and 6 Annales de l'Institut Pasteur, Vol, X. No. 5 and 9.

Zeitschrift f. Hygiene u. Infections Kr., Vol. XXII.

Dotschrift f. H yiene u. Infections $\mathrm{Kr}$., Vol.

\section{THE LIMITATIONS OF FORMALDEHYDE} IN DISINFECTION.

BY ELBERT W. ROCKWOOD, M.D.

PROFESSOR OF CHEMISTRY AND TOXICOLOGY, STATE UNIVERITY OF IOWA. IOWA CITY, IOWA.

If formaldehyde or formic aldehyde is to be extensively used as a disinfectant, as now seems probable, it is important that the medical profession should know its limitations as well as its power, since either the use of a worthless disinfectant or the improper use of a valuable one is but little removed from a criminal act. Recent experiments indicate that only under certain conditions can we be reasonably sure of destroying pathogenic organisms by means of form. aldehyde.

The compound is a gas of which the specific gravity is very nearly that of air. We should expect, therefore, that it would be slow to diffuse where air must be displaced. Pfuhl has shown (Zeit. für Hygiene, Vol. xxii, p. 339, Vol. xxIv, p. 289) that where it is present in sufficient quantities it sterilizes the surface of exposed objects, though its penetrating power is small. Similar results have been obtained by other experimenters. It is doubtful, then, whether it will replace other methods of disinfecting bedding, heavy clothing, etc. It has also been experimentally proven that a definite per cent. is necessary in the air to destroy bacterial life. Strüver (Zeit. für Hygiene, Vol: $\mathrm{xxv}, \mathrm{p} .356$ ) places this at $1.6 \mathrm{grams}$ of the gas in 1 cubic meter of space. He has shown too that the ordinary type of lamp used for the generation of formaldehyde by the action of platinum on the vapor of wood alcohol converts only about 8 or 9 per cent. of the latter into formaldehyde (loc. cit.). If a lamp is used for the production of the gas, it must be large enough to furnish the required per cent. to the air of the room. Moreover, since the formaldehyde has the same specific gravity as the air, it will diffuse at the same rate as the air is driven out of the room through the cracks and other openings. In order to be most effective not only must a large amount be generated, but this must be quickly done to prevent diffusion 
We can thus explain the failures which have been reported with small lamps. Trillat's method of generating the gas by driving it out of its solution by heating the latter under pressure in an autoclave, avoids the difficulty by setting free the gas very quickly.

Formic aldehyde $\left(\mathrm{CH}_{2} \mathrm{O}\right)$ is a gas under ordinary conditions, but it may be changed into a solid form. This is called paraformaldehyde or tri-oxymethylen. Its formula is $\left(\mathrm{CH}_{2} \mathrm{O}\right)_{3}$ and it may be considered as condensed formaldehyde. It is an imperfectly crystalline substance which is volatilized by heat. The density of the gas thus formed shows that the complex molecule has been decomposed and the simple one, $\mathrm{CH}_{2} \mathrm{O}$, formaldehyde, formed. This method of generating the gas directly from the solid, which takes place at a not high temperature, is likely to prove the most practical means of producing the disinfectant without the use of expensive apparatus as in Trillat's method.

\section{A GLANCE AT SOME RELATIONS OF DEN- TISTRY TO GENERAL MEDICINE.}

\footnotetext{
Presented to the Section on Stomatology at the Forty-eighth Annual Meeting of the American Medical Association, held at Philadelphia, Pa., June 1-4, 1897.

BY GEO. F. EAMES, M.D., D.D.S. BOS'TON, MAss.
}

The average dentist not only fails to recognize many indications which call for systemic treatment, but fails to see his need of such recognition. He still jumbles medical terms. His practice is largely empirical. He sees in part, but acts as one comprehending all; he gives advice to his fellow practitioners more freely than he would if he knew more.

If a new drug is advertised he tries it a few times, and immediately writes an article on it. He treats all forms of pyorrhea by scraping the teeth, but the deposit continues to accumulate, and the scraping is repeated. He treats sensitive dentine by the application of obtundents, and never dreams of prophylaxis or the correction of the underlying cause.

The teeth under certain conditions decay with great rapidity, and extra dollars are received for extra plugs inserted. The teeth are stained and eroded, only to be met with more plugging, and the mechanical removal of the stain.

During a persistent and.alarming hemorrhage from the socket of a tooth, the heart is allowed to go on thumping against the chest wall, thereby resisting the local styptic, and tending to increase the hemorrhage.

It is still true, in too many cases, that a tooth is extracted solely because the patient asks that it be done. Many dentists still ask the patient what kind of filling they will have inserted, little thinking that if in certain cases the request is complied with the dentist may be sued for malpractice.

The dental college with its required three years' course and Latin entrance examinations, still continues to let loose thousands of graduates whose spelling and use of ordinary English is excruciating, and whose understanding of the principles of pathology and medicine is practically nil. These go out to practice on humanity.

Shortly after I had begun to study dentistry, I was introduced by my preceptor to a very prominent minister who said, "so this young man is going to learn to haul teeth!" My preceptor wisely answered, "that, among other things." Those "other things" have engaged my attention ever since, and there are and ever will be, other things to follow, for if there is one characteristic of the marvelous advance which dentistry as a profession has made, it is the great number of new relations which have been discovered to exist, not only between it and other branches of medicine and surgery, but relations with the arts and sciences.

It has been said that the dentist's daily work is largely mechanical; many make it so; many see only the mechanical side of it; for example, an operator may be engaged in plugging a large distal cavity with gold, but may not realize the fact that in some cases of nervous susceptibility, the entire organism may sympathize with the local irritation.thus produced, to the extent of shock, or nervous collapse. What is the position of the mechanical operator in such a case? In his fail. ure to recognize the vital connection of the teeth with the human organism he has failed to discharge his full duty to the patient, and is guilty of criminal neglect.

A dentist may be called upon at any time to exercise his medical knowledge immediately, in cases of nervous spasm, hysteria, syncope, shock, collapse, foreign bodies swallowed, poisoning, hemorrhage, etc.; or in a more leisurely way in the consideration of questions relating to stomatitis, adenoid vegetations, malignant growths, operations during pregnancy, and the various inflammatory conditions of the dental pulp and pericementum.

But there are other fields which have not been so fully explored, and which are vital to the interests of our profession. Teeth are being lost at an alarming rate on account of the ravages of pyorrhea alveolaris, so called, and this fact alone calls for deep medical research, for a thorough physical examination, including the blood, the ealivary and renal secretions, followed by appropriate treatment.

In some of my recent cases the personal history has shown no indications of disease, when an examination of the secretions has shown conclusively the existence of abnormal conditions. This shows the necessity of a thorough examination, without regard to the patient's answers or knowledge of his condition.

In order to do this a most thorough study of the theory and practice of medicine is absolutely required. I can see no other way to meet these pathologic conditions in the mouth, believing as I do that they are the expression of some diathesis or other general disturbance. As medical specialists we are facing the serious problem of saving thousands of teeth which at the present time are being lost in spite of the great advances which our profession has made, and it rests with us, as members of the representative medical body of the United States, to use every means in our power to secure the requirement of a broader general and medical education as one of the essential conditions of graduation. If this be accomplished, the holder is sure of recognition in any medical organization, and the dental profession will stand for one of the most profound and philanthropic agents of the healing art.

Eye Protector-The Journal d'Hygiène of March 10 describes an extension for the eye-glasses of bicyclers, etc., made of celluloid or some similar substance, which clamps over the eye-glass and extends to enclose and fit tight over the entire orbital region, preventing the entrance of dust or wind, although ventilated at the side. 